\title{
Latest results on jet suppression and jet substructure in heavy-ion collisions with CMS
}

\author{
Jussi Viinikainen* on behalf of the CMS Collaboration. ${ }^{\dagger}$ \\ The University of Illinois at Chicago \\ E-mail: jussi.viinikainen@cern.ch
}

\begin{abstract}
CMS has performed a wide range of jet measurements in heavy-ion collisions. A selection of newest results in this field is presented. New results of the nuclear modification factor $R_{\mathrm{AA}}$ from $\mathrm{XeXe}$ collisions are compared to the previous results from $\mathrm{PbPb}$ collisions to study system size dependence of the energy loss. Energy loss in $\mathrm{PbPb}$ collisions is studied more differentially by measuring radial momentum distributions with inclusive jets. The dependence of parton flavor to energy loss is studied with photon-tagged jet shapes and by correlating tracks from reconstructed $D^{0}$ particles to the jet axis. Finally groomed jet substructure results for shared momentum fraction $z_{g}$ and jet mass $M_{g}$ are presented to go deeper into the details of energy loss mechanisms.
\end{abstract}

7th Annual Conference on Large Hadron Collider Physics - LHCP2019

20-25 May, 2019

Puebla, Mexico

\footnotetext{
${ }^{*}$ Speaker.

${ }^{\dagger}$ This work is supported by the grant DE-FG02-94ER40865 from the US Department of Energy.
} 


\section{Introduction}

Partons created in the initial hard scattering lose energy as they traverse the quark-gluon plasma created in ultra-relativistic heavy ion collisions [1]. These colored partons then fragment and hadronize to produce streams of final state particles called jets. As jets are products of colored partons that have interacted with the quark-gluon plasma, they are important probes to study the properties of this hot and dense state of matter.

Experimentally a jet is clustered from the final state particles using some jet reconstruction algorithm. One of the most popular ones, the anti- $k_{\mathrm{T}}$ algorithm [2], is used by the CMS experiment. When reconstructing jets in the heavy ion environment, one has to also take into account the huge combinatorial background in the events. Two alternative background subtraction methods are used for the presented results. The jet shape related studies use an iterative "noise/pedestal" subtraction technique, which is described in detail in Ref. [3]. The jet substructure studies featuring groomed jets apply a constituent subtraction algorithm [4] with the method described in Ref. [5] to estimate the background energy density.

We present recent results from the CMS collaboration studying the nuclear modification factors [6], jet shapes [7, 8, 9], shared momentum fractions [10] and groomed jet mass [11]. All the presented pp and $\mathrm{PbPb}$ results use datasets collected by the CMS experiment [12] in 2015 at collision energy $\sqrt{s_{\mathrm{NN}}}=5.02 \mathrm{TeV}$ corresponding to integrated luminosities of $404 \mu \mathrm{b}^{-1}$ for $\mathrm{PbPb}$ and $27.4 \mathrm{pb}^{-1}$ for pp. The XeXe dataset collected in 2017 at collision energy $\sqrt{s_{\mathrm{NN}}}=5.44 \mathrm{TeV}$ corresponding to an integrated luminosity of $3.42 \mu \mathrm{b}^{-1}$.

\section{Nuclear modification factor}

A traditional and still commonly used observable for energy loss studies is the nuclear modification factor $R_{\mathrm{AA}}$, which is defined as the ratio of the charged particle $p_{\mathrm{T}}$ spectra between nucleusnucleus (AA) collisions and proton-proton (pp) collisions, scaled by the number of binary nucleonnucleon interactions for the ion system $\left(\left\langle N_{\text {coll }}\right\rangle\right)$

$$
R_{\mathrm{AA}}=\frac{1}{\left\langle N_{\mathrm{coll}}\right\rangle} \frac{\mathrm{d} N^{\mathrm{AA}} / \mathrm{d} p_{\mathrm{T}}}{\mathrm{d} N^{\mathrm{pp}} / \mathrm{d} p_{\mathrm{T}}} .
$$

Recently the CMS collaboration has measured nuclear modification factors from XeXe collisions at $\sqrt{s_{\mathrm{NN}}}=5.44 \mathrm{TeV}$ [6]. The results for the $0-5 \%$ most central collisions compared to previous results for $\mathrm{PbPb}$ collisions at $\sqrt{s_{\mathrm{NN}}}=5.02 \mathrm{TeV}$ are shown of the left plot of Fig. 1. The nuclear modification factor for XeXe is denoted as $R_{\mathrm{AA}}^{*}$, since the measured reference pp spectrum does not exist for $\sqrt{s}=5.44 \mathrm{TeV}$. To construct the appropriate pp reference an extrapolation from the results at $\sqrt{s}=5.02 \mathrm{TeV}$ was used. It can be seen from this figure that in the region $p_{\mathrm{T}}>5 \mathrm{GeV}$, where the parton energy loss gives the dominant effect for $R_{\mathrm{AA}}, \mathrm{PbPb}$ collisions show more suppression than XeXe collisions. This can be understood as path length dependence, as collisions of $\mathrm{Pb}$ ions (radius of $6.6 \mathrm{fm}$ ) are likely to produce more medium than collisions of Xe ions (radius of $5.4 \mathrm{fm}$ ). This interpretation is supported by the right plot in Fig. 1. When the nuclear modification factor is plotted as a function of number of nucleons participating in the collisions, the suppression is similar in $\mathrm{PbPb}$ and $\mathrm{XeXe}$ collisions. 


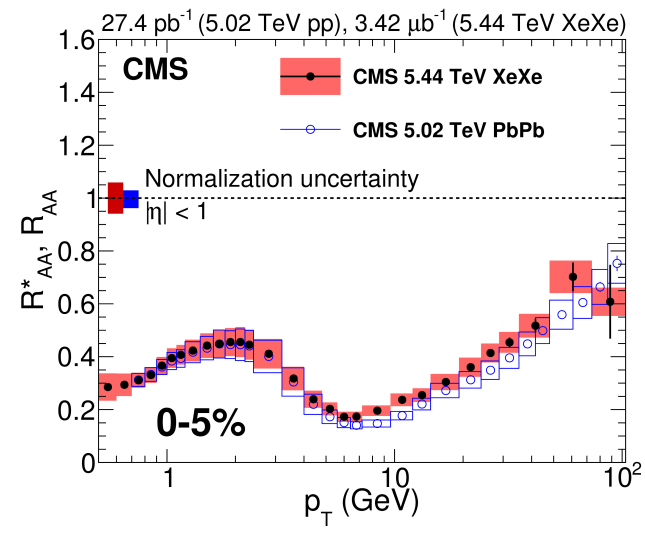

(a)

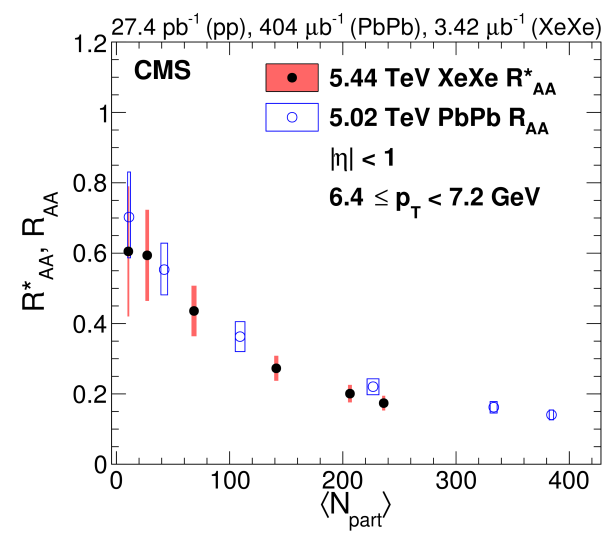

(b)

Figure 1: (Color online). Left: $R_{\mathrm{AA}}^{*}$ for XeXe and $R_{\mathrm{AA}} \mathrm{PbPb}$ collisions for the $0-5 \%$ centrality bin. Right: $R_{\mathrm{AA}}^{*}$ for $\mathrm{XeXe}$ and $R_{\mathrm{AA}} \mathrm{PbPb}$ collisions as a function of number of participating nucleons $\left(N_{\text {part }}\right)$. Figures from [6].

\section{Jet shapes}

Jet energy loss in the quark-gluon plasma can be studied more differentially by looking at the distribution of tracks with respect to the jet axis as a function of the angular distance $\Delta r=$ $\sqrt{\Delta \eta^{2}+\Delta \varphi^{2}}$. The radial momentum distribution of the jet $P(\Delta r)$ is defined to be the momentum weighted distribution of the tracks around the jet axis

$$
P(\Delta r)=\frac{1}{\delta r} \frac{1}{N_{\text {jets }}} \sum_{\text {jets }}\left(\sum_{\text {track } \in\left(r_{a}, r_{b}\right)} p_{\mathrm{T}}^{\text {track }}\right) .
$$

The jet shape $\rho(\Delta r)$ is the radial momentum distribution normalized to one over the range that is studied

$$
\rho(\Delta r)=\frac{1}{\delta r} \frac{1}{N_{\text {jets }}} \sum_{\text {jets }} \frac{\sum_{\text {track } \in\left(r_{a}, r_{b}\right)} p_{\mathrm{T}}^{\text {track }}}{p_{\mathrm{T}}^{\text {jets }}} .
$$

CMS has measured the radial momentum distributions for inclusive jets with transverse momentum above $120 \mathrm{GeV}$ [7]. The top row in Fig.2 shows the distribution in pp collisions while the different centrality bins for $\mathrm{PbPb}$ collisions are shown in the middle row. Concentrating on the ratio in the bottom row, the modification in the $\mathrm{PbPb}$ momentum distribution with respect to the pp collisions can clearly be seen. In the most peripheral bin the modifications are modest, but they become more and more clear towards the central collisions. It can be seen that there is an enhancement of low- $p_{\mathrm{T}}$ particles and a depletion of high- $p_{\mathrm{T}}$ particles with respect to pp measurements at large radius. This tells us that the energy lost at high transverse momenta due to interactions with the quark-gluon plasma reappears in form of low- $p_{\mathrm{T}}$ particles far away from the jet axis.

Requiring a back-to-back photon together with a jet helps to better constrain initial kinematics of the scattering process $[13,14,15]$ and also biases the jet selection towards quark jets [16], which gives additional insight on the parton flavor dependence of the energy loss [17, 18]. CMS has made photon-tagged jet shape measurements with $p_{\mathrm{T}}^{\text {jet }}>30 \mathrm{GeV}, p_{\mathrm{T}}^{\gamma}>60 \mathrm{GeV}$ and separation between 


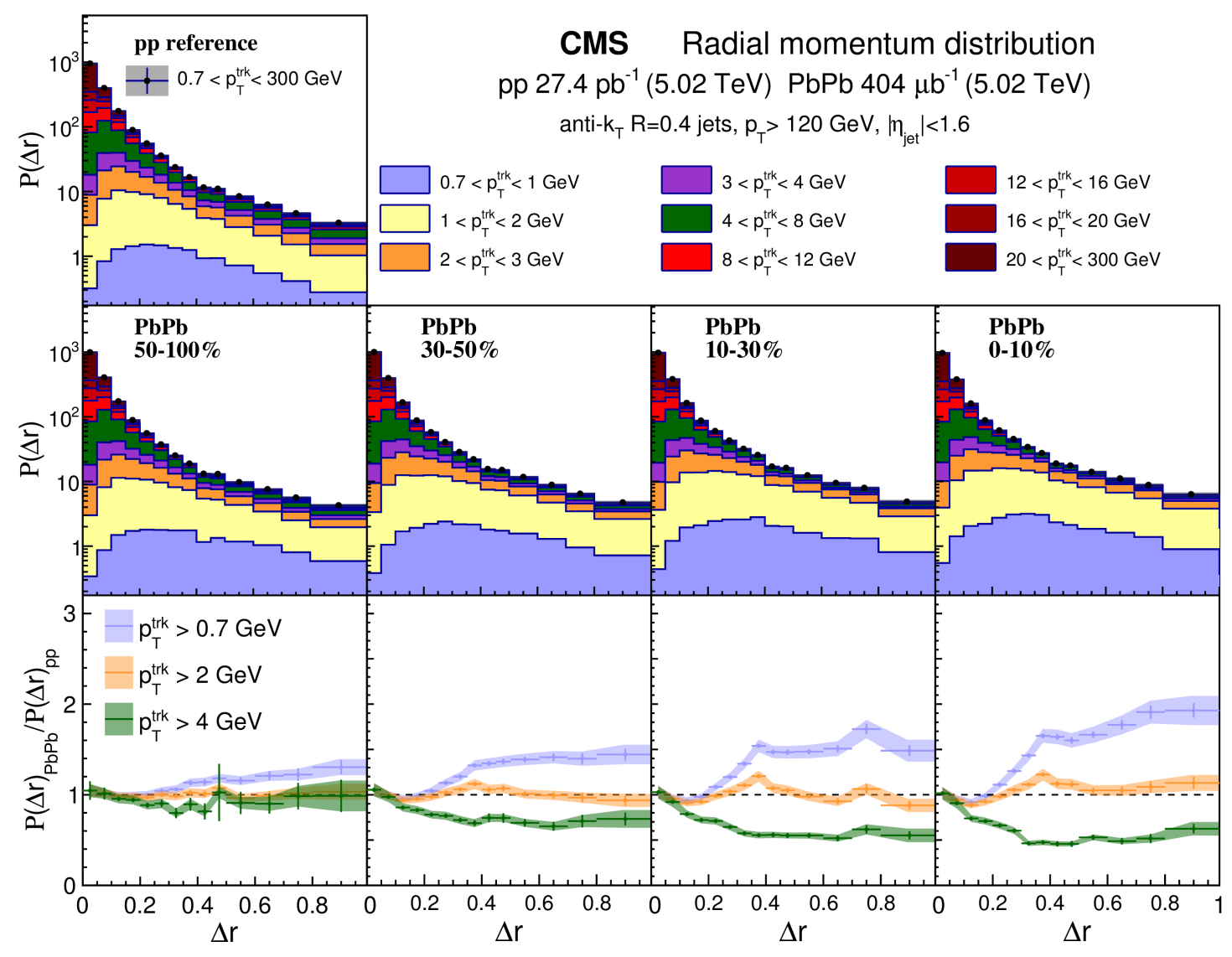

Figure 2: (Color online). Jet radial momentum distribution for inclusive jets above $120 \mathrm{GeV}$ from pp collisions (top row) and $\mathrm{PbPb}$ collisions (middle row). The contributions to the total jet shape from different track $p_{\mathrm{T}}$ bins are drawn as stacked histograms. The bottom row shows the ratio between $\mathrm{PbPb}$ and $\mathrm{pp}$ in three track $p_{\mathrm{T}}$ regions. Figure from [7].

jet and photon $\Delta \varphi>7 \pi / 8$ [8]. Looking at the ratios in the bottom row of plots in Fig. 3, a similar effect as for the inclusive jets can be seen. There is a small depletion of tracks close to the jet axis that allows for the large enhancement far from it.

CMS has also made jet shape related measurements featuring heavy quarks [9]. The charm production in the quark-gluon plasma and heavy flavor energy loss have been studied by measuring density profiles of reconstructed $D^{0}$ mesons around the jet axis. This study uses jets above $p_{\mathrm{T}}^{\text {jet }}>$ $60 \mathrm{GeV}$. The radial track density profile for $D^{0}$ mesons in the range $4<p_{\mathrm{T}}^{\mathrm{D}}<20 \mathrm{GeV}$ is shown on the left side of Fig. 4 and in the range $p_{\mathrm{T}}^{\mathrm{D}}>20 \mathrm{GeV}$ on the right side of that figure. The lower $p_{\mathrm{T}}^{\mathrm{D}}$ selection shows a hint of shape broadening towards larger radius, which could be caused by a charm diffusion inside the medium [19, 20,21]. No significant modifications are seen in the higher $p_{\mathrm{T}}^{\mathrm{D}}$ selection. However, it should be noted that the uncertainties for this measurement are still large and more data are needed before stronger conclusions can be made. 


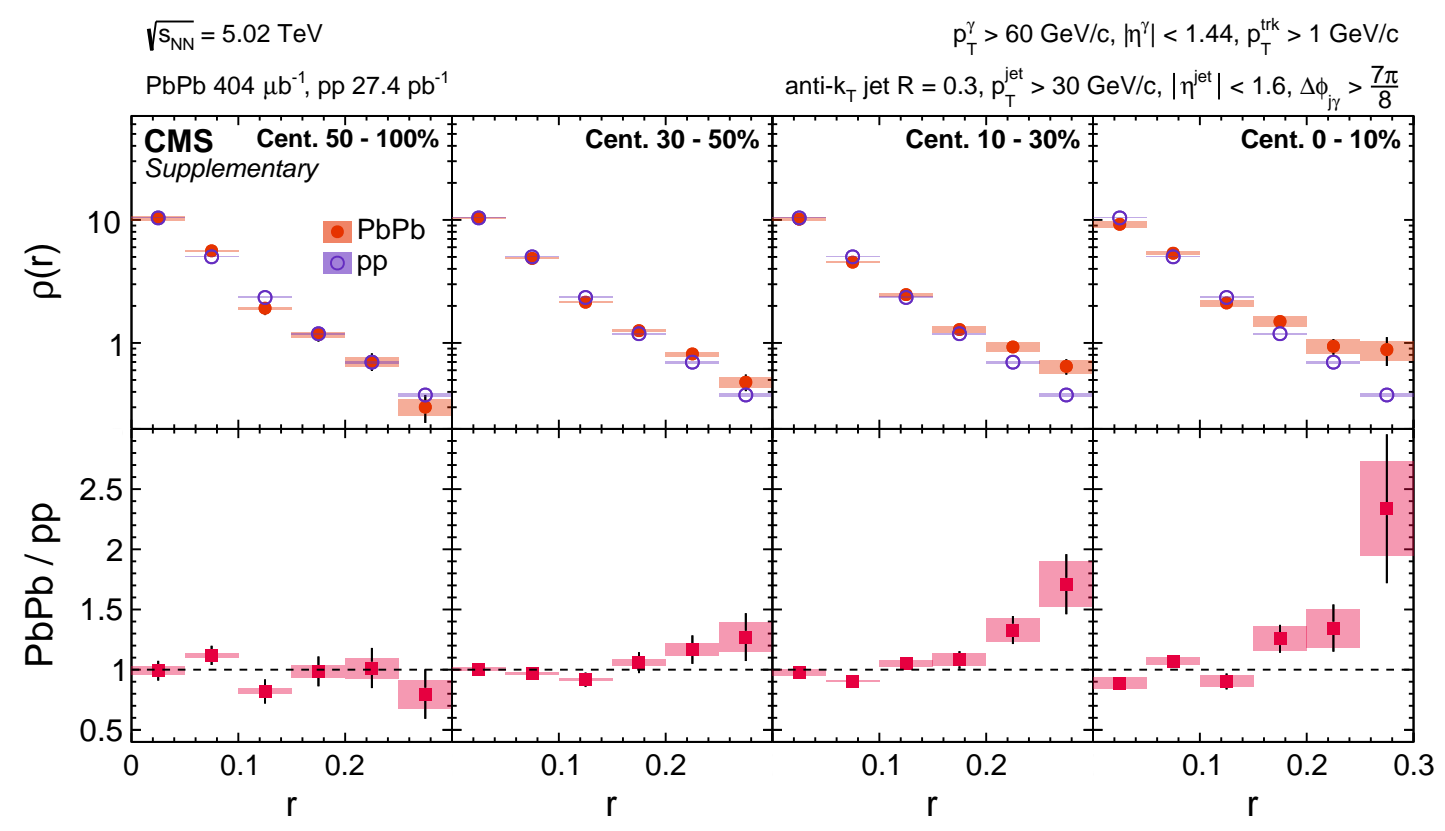

Figure 3: (Color online). Jet shape from $\gamma$-tagged jets. The top row shows the comparison of jet shape distribution between pp collisions and different centrality bins in $\mathrm{PbPb}$ collisions. The ratio between $\mathrm{PbPb}$ and pp jet shapes is shown in the bottom row. Figure from [8].

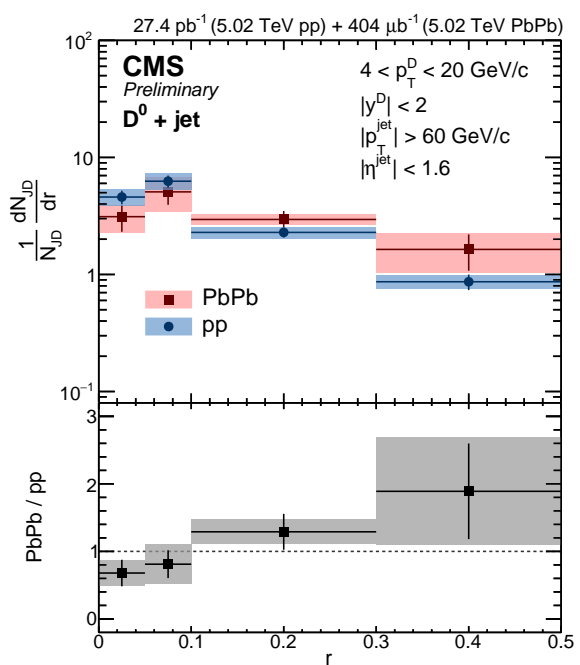

(a)

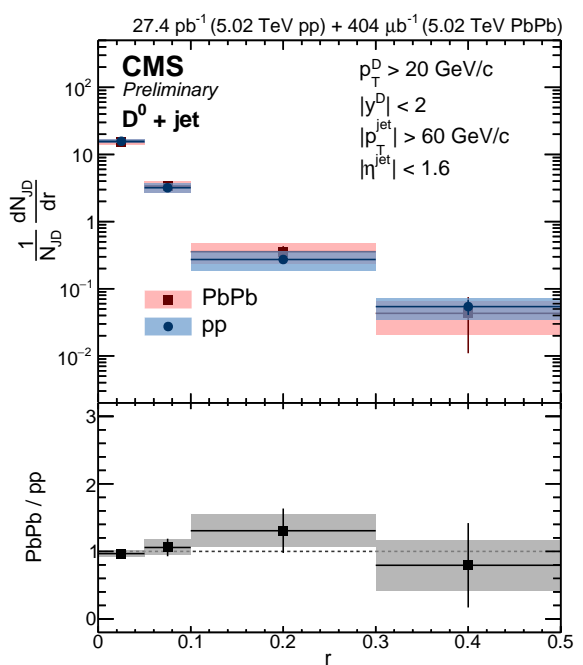

(b)

Figure 4: (Color online). Left: Radial track density profile for tracks from reconstructed $D^{0}$-mesons correlated to the jet axis for $4<p_{\mathrm{T}}^{\mathrm{D}}<20 \mathrm{GeV}$. Right: Radial track density profile for tracks from reconstructed $D^{0}$-mesons correlated to the jet axis for $p_{\mathrm{T}}^{\mathrm{D}}>20 \mathrm{GeV}$. Figures from [9]. 


\section{Groomed jet observables}

To concentrate only to the hard core of the jet, grooming algorithms [22, 23, 24] can be used to remove soft particles within the jet cone. The specific grooming algorithm used in the results presented here is the soft drop algorithm [23, 25]. In this method the jet is first reconstructed with the anti- $k_{\mathrm{T}}$ algorithm [2], and then reclustered with the Cambridge-Aachen scheme [26] to create a pairwise tree of subjets. Then, the jet is declustered by dropping the softer of the two branches in each step if the soft drop condition

$$
z_{g}=\frac{\min \left(p_{\mathrm{T}, i}, p_{\mathrm{T}, j}\right)}{p_{\mathrm{T}, i}+p_{\mathrm{T}, j}}>z_{\text {cut }}\left(\frac{\Delta R_{i j}}{R_{0}}\right)^{\beta}
$$

is not satisfied. In this equation $p_{\mathrm{T}, i}$ and $p_{\mathrm{T}, j}$ denote the transverse momenta of the two subjets, $R_{i j}$ is the angular separation between the subjets in $\varphi-\eta$ plane, $R_{0}$ is the $\mathrm{R}$ parameter used in the anti$k_{T}$ algorithm, and $z_{\text {cut }}$ and $\beta$ are tunable parameters. Two different parameter sets are considered for the measurements presented here: $z_{\text {cut }}=0.1$ with $\beta=0$, denoted as $(0.1,0)$ soft drop setting, and $z_{\text {cut }}=0.5$ with $\beta=1.5$, denoted as $(0.5,1.5)$ soft drop setting. The first parameter set has good theoretical properties, as it is largely insensitive to higher order QCD corrections, such as multiple emissions. The second set, on the other hand, has better experimental properties, since it reduces the impact of the underlying event by imposing stricter soft drop condition on subjets with larger opening angle, thus being more focused on the core of the jet. An additional cut $R_{i j}>0.1$ is used for both parameter sets to take into account the fact that subjets with small opening angle cannot often be distinctly resolved.

The variable $z_{g}$ corresponds to the QCD splitting function in pp collisions [27] and can help to probe the role of color coherence effects in $\mathrm{PbPb}$ collisions [28]. If the partons forming the two subjets in the quark-gluon plasma act as a single coherent emitter, the two subjets will be equally modified leaving $z_{g}$ intact [29]. However, if the partons act as a decoherent emitter, two subjets can be modified differently and consequently also $z_{g}$ will be altered. Other phenomena to which $z_{g}$ is sensitive include semi-hard medium-induced gluon radiation [30], modifications of the initial parton splitting [31], and the medium response [32].

The $z_{g}$ distributions have been measured by $\mathrm{CMS}$ in $\mathrm{PbPb}$ collisions and compared to results in pp collisions smeared to match the resolution in $\mathrm{PbPb}$ events [10]. The different centrality selections for jets in $160<p_{\mathrm{T}}^{\text {jet }}<180 \mathrm{GeV}$ bin for soft drop settings $(0.1,0)$ are compared to smeared pp results in the left side of Fig. 5. It can be seen from the ratio presented at the bottom of the figure that the peripheral $\mathrm{PbPb}$ distributions are consistent with $\mathrm{pp}$, but the division to two subjets becomes more imbalanced for central $\mathrm{PbPb}$ collisions. The right plot in Fig. 5 shows a selection of different $p_{\mathrm{T}}^{\mathrm{jet}}$ intervals for the most central bin, together with a comparison to predictions from JEWEL event generator [33, 34] as well as to several theory calculations [32, 31, 35].

The ability of the quark-gluon plasma to resolve traversing partons depends also on the opening angle of the splitting [30,31]. The opening angle effects can be assessed using the invariant mass of the two-subjet system, called groomed jet mass $M_{g}$. The results for the groomed jet mass are presented normalized by the jet transverse momentum as the invariant mass scales with $p_{\mathrm{T}}^{\text {jet }}$ in the vacuum [24]. This normalization also leads to partial cancellation of systematic uncertainties. 


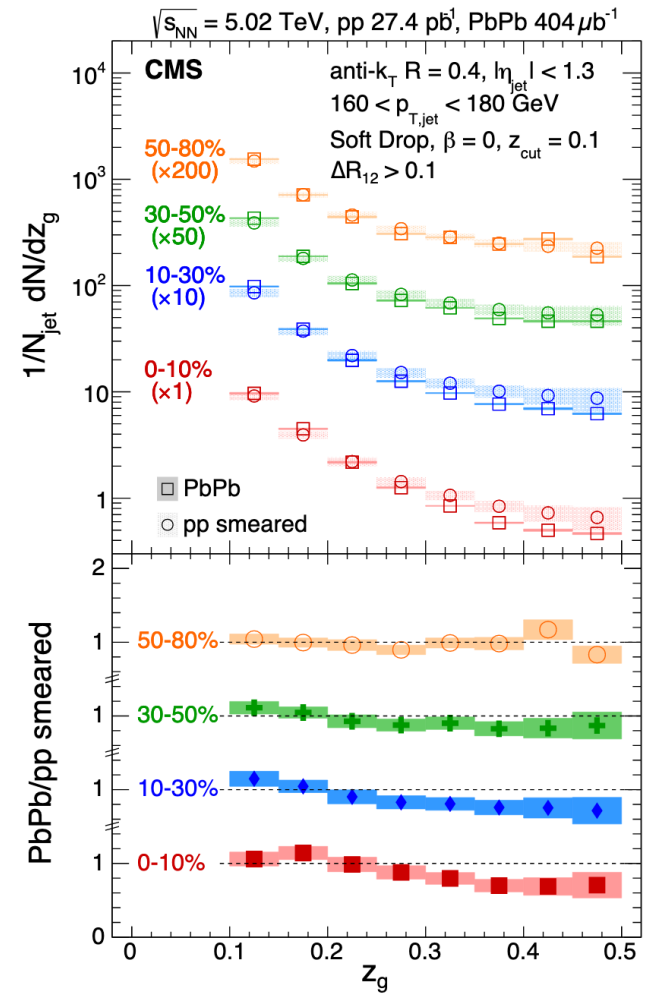

(a)

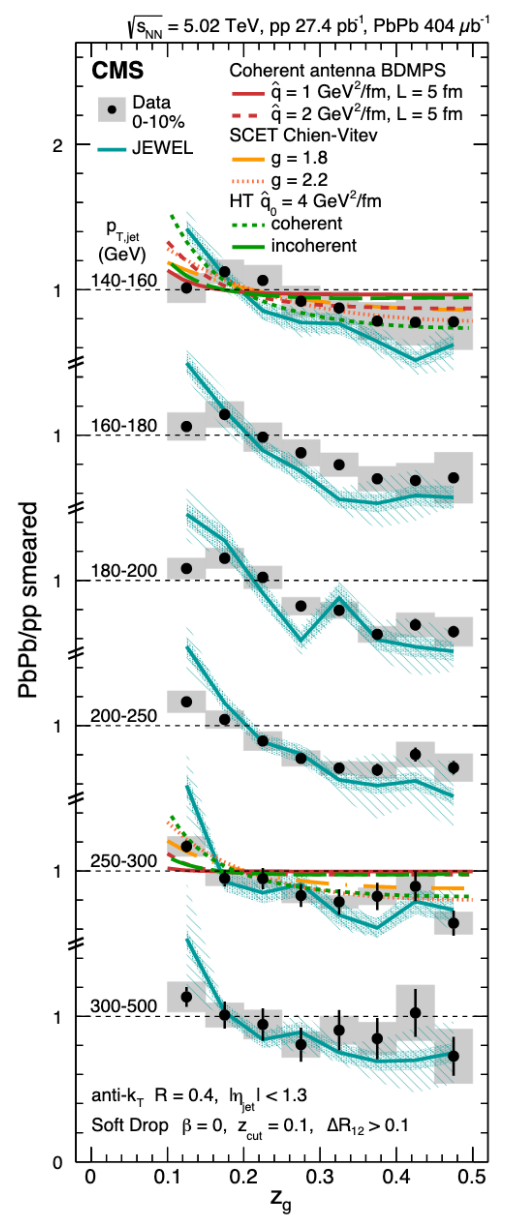

(b)

Figure 5: (Color online). Left: The $z_{g}$ distribution in different centrality bins in $\mathrm{PbPb}$ collisions in the bin $160<p_{\mathrm{T}}^{\text {jet }}<180 \mathrm{GeV}$ compared to results from pp collisions smeared to match the $\mathrm{PbPb}$ resolution. The error bars show statistical and the shaded areas systemtic uncertainty. Right: Ratios of $z_{g}$ distribution between the $10 \%$ most central $\mathrm{PbPb}$ collisions and smeared pp collisions in several diffetent jet $p_{\mathrm{T}}$ ranges. The results are compared to various theoratical calculations $[32,31,35,33]$. Soft drop setting $(0.1,0)$ is used for both figures. Figures from [10].

The CMS measurement of $M_{g} / p_{\mathrm{T}}^{\text {jet }}$ [11] using $(0.1,0)$ soft drop settings in the $160<p_{\mathrm{T}}^{\text {jet }}<$ $180 \mathrm{GeV}$ bin for $\mathrm{PbPb}$ collisions in different centrality bins is compared to smeared pp results in the left and middle panels of Fig. 6. The ratio of the most central $\mathrm{PbPb}$ bin to the smeared $\mathrm{pp}$ sample in several different different jet $p_{\mathrm{T}}$ selections is compared to predictions from JEWEL and Q-Pythia [36] generators in the right panel of this figure. The same three panels for $(0.5,1.5) \mathrm{soft}$ drop settings are presented in Fig. 7. No modifications between $\mathrm{PbPb}$ and pp collisions are seen with $(0.5,1.5)$ soft drop settings, which concentrate on the core of the jet. For the $(0.1,0)$ soft drop settings there is a hint of enhancement for the large mass region in the most central bin, but otherwise there are no significant modifications. From the models compared to data here, none can describe the jet mass measurements for the two grooming settings simultaneously. 


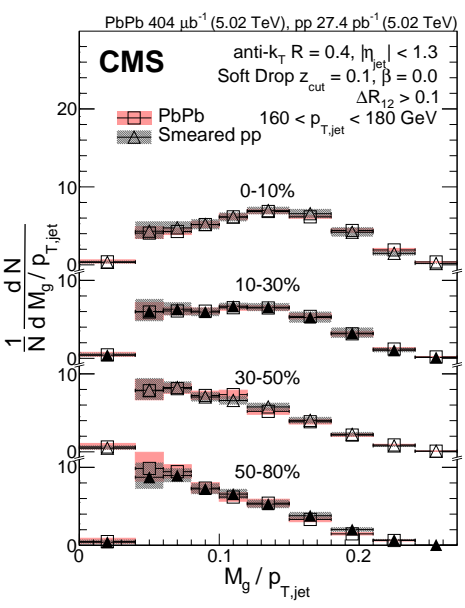

(a)

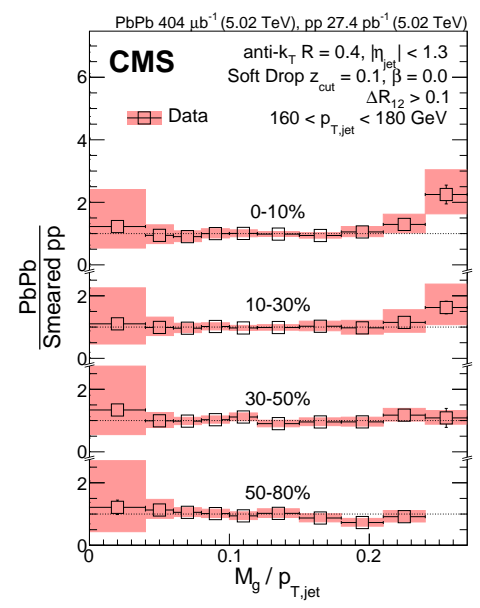

(b)

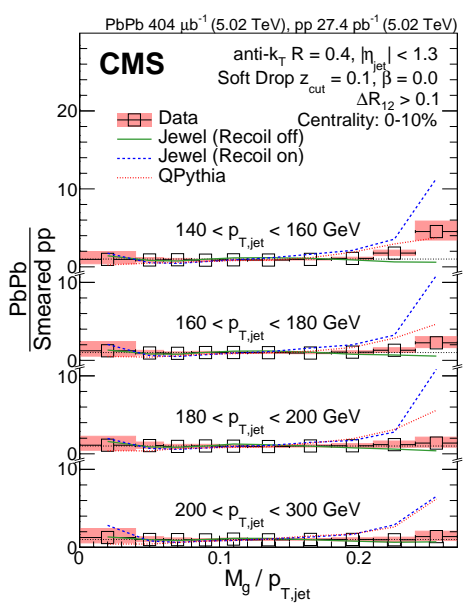

(c)

Figure 6: (Color online). Left: The centrality dependence of $M_{g} / p_{\mathrm{T}}^{\text {jet }}$ for $\mathrm{PbPb}$ collisions in the $160<p_{\mathrm{T}}^{\text {jet }}<$ $180 \mathrm{GeV}$ bin using $(0.1,0)$ soft drop settings compared to results from pp collisions smeared to match the $\mathrm{PbPb}$ resolution. Middle: The ratio of $\mathrm{PbPb}$ jet mass over smeared pp jet mass. The error bars show statistical uncertainty and the filled area systematic uncertainties. Right: The ratio of $\mathrm{PbPb}$ jet mass over smeared $\mathrm{pp}$ jet mass in the 0-10\% bin for several jet $p_{\mathrm{T}}$ intervals. The data are compared to smeared predictions from JEWEL and Q-Pythia. Figures from [11].

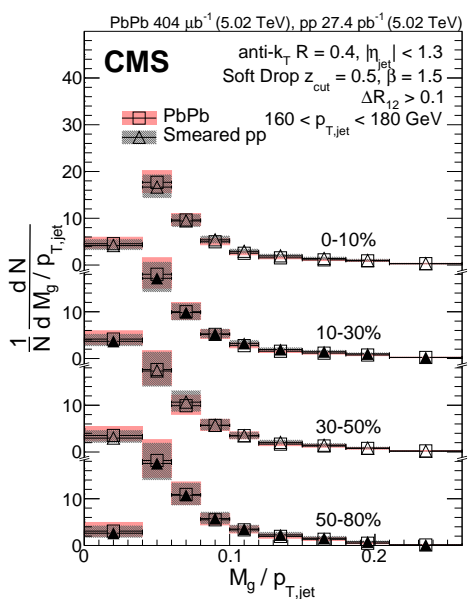

(a)

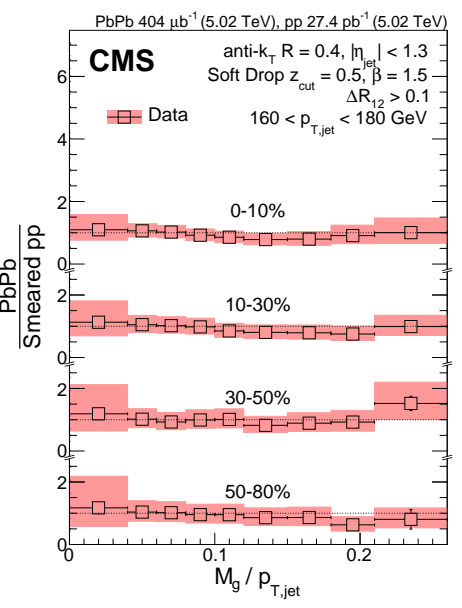

(b)

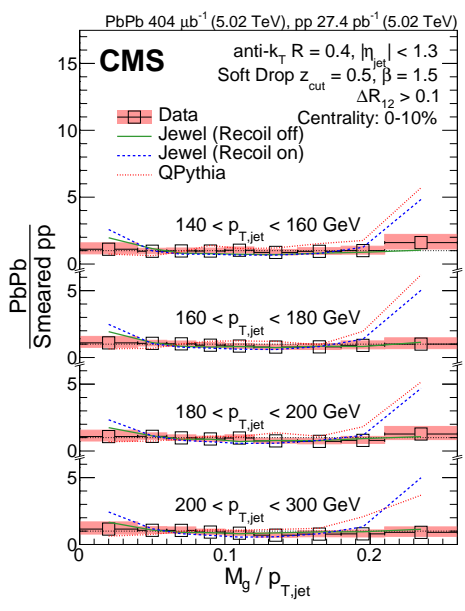

(c)

Figure 7: (Color online). Left: The centrality dependence of $M_{g} / p_{\mathrm{T}}^{\text {jet }}$ for $\mathrm{PbPb}$ collisions in the $160<$ $p_{\mathrm{T}}^{\text {jet }}<180 \mathrm{GeV}$ bin using $(0.5,1.5)$ soft drop settings compared to results from pp collisions smeared to match the $\mathrm{PbPb}$ resolution. Middle: The ratio of $\mathrm{PbPb}$ jet mass over smeared pp jet mass. The error bars show statistical uncertainty and the filled area systematic uncertainties. Right: The ratio of $\mathrm{PbPb}$ jet mass over smeared pp jet mass in the $0-10 \%$ bin for several jet $p_{\mathrm{T}}$ intervals. The data are compared to smeared predictions from JEWEL and Q-Pythia. Figures from [11]. 


\section{Summary}

CMS has recently published several studies on jet suppression and jet substructure modifications in quark-gluon plasma. The recent results for nuclear modification factors in $\sqrt{s_{\mathrm{NN}}}=$ 5.44 $\mathrm{TeV} \mathrm{XeXe}$ collisions show that the suppression is smaller than for $\mathrm{PbPb}$ in the same centrality class, reflecting a larger path length inside the QGP for partons in a large system. However, for the same number of participating nucleons, the suppression strength becomes comparable between these two systems.

Jet shapes have been measured in $\mathrm{PbPb}$ collisions at $\sqrt{s_{\mathrm{NN}}}=5.02 \mathrm{TeV}$ and in pp collisions at $\sqrt{s}=5.02 \mathrm{TeV}$. The radial momentum distribution for inclusive jets shows broadening of the shape in $\mathrm{PbPb}$ compared to pp collisions. It is also observed that if we look only at high- $p_{\mathrm{T}}$ tracks, instead of enhancement at large $\Delta r$ the measurements show a depletion.

The jet shape for photon-tagged jets shows overall similar trends as the momentum distribution for inclusive jets. However, broadening of the shape is also seen when correlating $D^{0}$ mesons with the jet axis for the bin $4<p_{\mathrm{T}}^{\mathrm{D}}<20 \mathrm{GeV}$, while a narrowing is already seen for this track $p_{\mathrm{T}}$ bin for inclusive jets. This could be a hint of charm diffusion in the medium, but more data are needed to shrink the error bars before strong conclusions could be made here.

The results for a shared momentum fraction $z_{g}$ show the groomed two-subjet system becoming more imbalanced in central $\mathrm{PbPb}$ collisions compared to pp collisions with grooming settings $(0.1,0)$. For the same grooming settings, the jet $p_{\mathrm{T}}$ normalized groomed jet mass $M_{g}$ shows enhancement at large mass region in the $0-10 \%$ centrality in $\mathrm{PbPb}$ collisions compared to pp collisions. No modifications in $M_{g} / p_{\mathrm{T}}^{\text {jet }}$ are seen in other centrality bins or with grooming settings $(0.5,1.5)$, which preferably select two-subjet systems with a small opening angle.

\section{References}

[1] J. D. Björken, Energy Loss of Energetic Partons in Quark - Gluon Plasma: Possible Extinction of High $p_{\mathrm{T}}$ Jets in Hadron - Hadron Collisions, FERMILAB-PUB-82-059-THY (1982) .

[2] M. Cacciari, G. P. Salam and G. Soyez, The anti- $k_{\mathrm{T}}$ jet clustering algorithm, JHEP 04 (2008) 063 [0802.1189].

[3] O. Kodolova, I. Vardanyan, A. Nikitenko and A. Oulianov, The performance of the jet identification and reconstruction in heavy ions collisions with CMS detector, Eur. Phys. J. C50 (2007) 117.

[4] P. Berta, M. Spousta, D. W. Miller and R. Leitner, Particle-level pileup subtraction for jets and jet shapes, JHEP 06 (2014) 092 [1403.3108].

[5] G. Soyez, G. P. Salam, J. Kim, S. Dutta and M. Cacciari, Pileup subtraction for jet shapes, Phys. Rev. Lett. 110 (2013) 162001 [1211.2811].

[6] CMS collaboration, Charged-particle nuclear modification factors in XeXe collisions at $\sqrt{s_{\mathrm{NN}}}=5.44 \mathrm{TeV}, \operatorname{JHEP} 10$ (2018) 138 [1809.00201].

[7] CMS collaboration, Jet properties in PbPb and pp collisions at $\sqrt{s_{\mathrm{NN}}}=5.02 \mathrm{TeV}$, JHEP 05 (2018) $006[1803.00042]$.

[8] CMS collaboration, Jet shapes of isolated photon-tagged jets in PbPb and pp collisions at $\sqrt{s_{\mathrm{NN}}}=5.02$ TeV, Phys. Rev. Lett. 122 (2019) 152001 [1809.08602]. 
[9] CMS collaboration, Measurement of the radial profile of $D^{0}$ mesons in jets produced in pp and $\mathrm{PbPb}$ collisions at 5.02 TeV, CMS-PAS-HIN-18-007 (2018) .

[10] CMS collaboration, Measurement of the splitting function in pp and PbPb collisions at $\sqrt{s_{\mathrm{NN}}}=5.02$ TeV, Phys. Rev. Lett. 120 (2018) 142302 [1708.09429].

[11] CMS collaboration, Measurement of the groomed jet mass in PbPb and pp collisions at $\sqrt{s_{\mathrm{NN}}}=5.02 \mathrm{TeV}$, JHEP 10 (2018) 161 [1805.05145].

[12] CMS collaboration, The CMS Experiment at the CERN LHC, JINST 3 (2008) S08004.

[13] X.-N. Wang, Z. Huang and I. Sarcevic, Jet quenching in the opposite direction of a tagged photon in high-energy heavy ion collisions, Phys. Rev. Lett. 77 (1996) 231 [hep-ph/9605213].

[14] X.-N. Wang and Z. Huang, Study medium induced parton energy loss in gamma + jet events of high-energy heavy ion collisions, Phys. Rev. C55 (1997) 3047 [hep-ph/9701227].

[15] W. Dai, I. Vitev and B.-W. Zhang, Momentum imbalance of isolated photon-tagged jet production at RHIC and LHC, Phys. Rev. Lett. 110 (2013) 142001 [1207. 5177].

[16] R. B. Neufeld, I. Vitev and B. W. Zhang, The Physics of $Z^{0} / \gamma^{*}$-tagged jets at the LHC, Phys. Rev. C83 (2011) 034902 [1 006.2389$]$.

[17] J. Casalderrey-Solana, D. C. Gulhan, J. G. Milhano, D. Pablos and K. Rajagopal, Predictions for Boson-Jet Observables and Fragmentation Function Ratios from a Hybrid Strong/Weak Coupling Model for Jet Quenching, JHEP 03 (2016) 053 [1508.00815].

[18] Z.-B. Kang, I. Vitev and H. Xing, Vector-boson-tagged jet production in heavy ion collisions at energies available at the CERN Large Hadron Collider, Phys. Rev. C96 (2017) 014912 [1702.07276].

[19] M. Nahrgang, J. Aichelin, P. B. Gossiaux and K. Werner, Azimuthal correlations of heavy quarks in $P b+P b$ collisions at $\sqrt{s}=2.76$ TeV at the CERN Large Hadron Collider, Phys. Rev. C90 (2014) 024907 [1305.3823].

[20] S. Cao, G.-Y. Qin and S. A. Bass, Modeling of heavy-flavor pair correlations in Au-Au collisions at 200A GeV at the BNL Relativistic Heavy Ion Collider, Phys. Rev. C92 (2015) 054909 [1505.01869].

[21] R. Hambrock and W. A. Horowitz, AdS/CFT predictions for azimuthal and momentum correlations of $b \bar{b}$ pairs in heavy ion collisions, Nucl. Part. Phys. Proc. 289-290 (2017) 233 [1 703 . 05845 ].

[22] J. M. Butterworth, A. R. Davison, M. Rubin and G. P. Salam, Jet substructure as a new Higgs search channel at the LHC, Phys. Rev. Lett. 100 (2008) 242001 [0802 . 2470 ].

[23] A. J. Larkoski, S. Marzani, G. Soyez and J. Thaler, Soft Drop, JHEP 05 (2014) 146 [1402 . 265 7].

[24] M. Dasgupta, A. Fregoso, S. Marzani and G. P. Salam, Towards an understanding of jet substructure, JHEP 09 (2013) 029 [1307.0007].

[25] A. J. Larkoski, S. Marzani and J. Thaler, Sudakov Safety in Perturbative QCD, Phys. Rev. D91 (2015) $111501[1502.01719]$.

[26] Y. L. Dokshitzer, G. D. Leder, S. Moretti and B. R. Webber, Better jet clustering algorithms, JHEP 08 (1997) 001 [hep-ph/9707323].

[27] A. Larkoski, S. Marzani, J. Thaler, A. Tripathee and W. Xue, Exposing the QCD Splitting Function with CMS Open Data, Phys. Rev. Lett. 119 (2017) 132003 [170 405066 ]. 
[28] Y. Mehtar-Tani and K. Tywoniuk, Jet (de)coherence in Pb-Pb collisions at the LHC, Phys. Lett. B744 (2015) 284 [1401.8293].

[29] J. Casalderrey-Solana, Y. Mehtar-Tani, C. A. Salgado and K. Tywoniuk, New picture of jet quenching dictated by color coherence, Phys. Lett. B725 (2013) 357 [1210 . 7765].

[30] Y. Mehtar-Tani and K. Tywoniuk, Groomed jets in heavy-ion collisions: sensitivity to medium-induced bremsstrahlung, JHEP 04 (2017) 125 [1610 . 08930 ].

[31] Y.-T. Chien and I. Vitev, Probing the Hardest Branching within Jets in Heavy-Ion Collisions, Phys. Rev. Lett. 119 (2017) 112301 [1608.07283].

[32] Y. Mehtar-Tani and K. Tywoniuk, Groomed jets in heavy-ion collisions: sensitivity to medium-induced bremsstrahlung, JHEP 04 (2017) 125 [1610.08930].

[33] G. Milhano, U. A. Wiedemann and K. C. Zapp, Sensitivity of jet substructure to jet-induced medium response, Phys. Lett. B779 (2018) 409 [1707. 04142$].$

[34] R. Kunnawalkam Elayavalli and K. C. Zapp, Medium response in JEWEL and its impact on jet shape observables in heavy ion collisions, JHEP 07 (2017) 141 [1707. 01539].

[35] N.-B. Chang, S. Cao and G.-Y. Qin, Probing medium-induced jet splitting and energy loss in heavy-ion collisions, Phys. Lett. B781 (2018) 423 [1707.03767].

[36] N. Armesto, L. Cunqueiro and C. A. Salgado, Q-PYTHIA: A Medium-modified implementation of final state radiation, Eur. Phys. J. C63 (2009) 679 [0 907 . 1014 ]. 